\title{
28 Research Soure \\ Specific serodiagnosis of animal brucellosis based on a recombinant multiepitope protein antigen
}

\section{Dehui Yin}

Xuzhou Medical University https://orcid.org/0000-0002-7164-9320

\section{Qiongqiong Bai}

Xuzhou Medical University

\section{Han Li}

Jilin University

Jinpeng Zhang ( $\nabla$ xiaopangpeng@126.com )

Xuzhou Medical University

\section{Research article}

Keywords: Brucellosis, Diagnosis, Recombinant multiepitope protein

Posted Date: September 8th, 2020

DOl: https://doi.org/10.21203/rs.3.rs-55889/v1

License: (c) (i) This work is licensed under a Creative Commons Attribution 4.0 International License. Read Full License 


\section{Abstract}

Background: Brucellosis is a vital zoonotic infectious disease that will cause important public health problems and endanger the development of animal husbandry in endemic areas, causing huge losses of personal property. Early diagnosis of sick animals is a crucial way to reduce the incidence of brucellosis.

Results: In this study, we designed a recombinant multiepitope protein (rMEP) as a serum diagnostic antigen for brucellosis. An indirect enzyme-linked immunosorbent assay (ELISA) was used to assess the new rMEP, 159 goat and 153 bovine serum samples were measured, including brucellosis and nonbrucellosis. To better observe the effectiveness of rMEP, we performed a receiver operating characteristic (ROC) analysis. The results of 159 goat serum evaluations showed that the area under the ROC curve(AUC) was 0.9976 and compared with SAT and RBPT, the positive and negative diagnostic accuracy of the ELISA was $98.92 \%(92 / 93)$ and $96.97 \%(64 / 66)$, respectively. 153 bovine serum samples evaluations showed the AUC was 0.9974 and compared with SAT and RBPT, the positive and negative diagnostic accuracy of the ELISA was $98.65 \%(73 / 74)$ and $96.20 \%(76 / 79)$, respectively.

Conclusions: The results show that rMEP as a protein antigen can be used to diagnose brucellosis with highly accurate both in goat and bovine.

\section{Background}

Brucellosis is a chronic zoonotic disease of great public health significance caused by Gram-negative, facultative, intracellular bacteria of the genus Brucella[1]. There are 6 classic types in the genus Brucella, namely, $B$. abortus, B. melltensis, B. ovis, B. Neotormae, $B$. suis, and B. canis[2]. The disease causes animals to miscarry and become infertile, it can also be transmitted to humans by touching infected animals or eating infected food, such as dairy products. B.melitensis, B.bovis, and B.suis are three major types of Brucella that are prevalent in China, of these, brucellosis in sheep is most common, followed by Brucella in cattle, all of these are the most common sources of brucellosis in humans[3]. Also, infected animals also develop orchitis, arthritis, and other symptoms. Animals have varying degrees of susceptibility to the disease. In recent years, the number of human and animal infections caused by Brucella has been increasing, which seriously threatens people's health and the development of animal husbandry. Over 0.5 million new human brucellosis cases are reported every year worldwide[4, 5]. In China, the number of sheep and goat populations infected with Brucella is on the rise and is highly endemic in some areas[6]. Because brucellosis is mostly a chronic disease, and there are still some problems with diagnostic methods, it is still an important public health problem in many countries, especially in backward rural areas[4]. Following Brucella is infected, treatment is difficult, so the emphasis on brucellosis is prevention and control. Conventional live, attenuated strains $B$. melitensis and $B$. abortion B19 are currently the most commonly used vaccines[7]. However, these vaccines also have some disadvantages, such as the possibility of miscarriage in pregnant animals[8]. Also, vaccination often complicates the distinction between naturally infected and vaccinated animals, which is not conducive to 
the diagnosis and control of brucellosis[9]. So, a timely and accurate diagnosis is important for the prevention and control of the disease.

There are many methods available for the diagnosis of brucellosis in animals, such as serological diagnosis, etiological diagnosis, molecular biology diagnosis, etc.[10]. Although the bacteriological diagnosis is the gold standard, it is not only time consuming and laborious, but also easily causes operator infection. Molecular biology pathogen detection technology has the characteristics of safety, reliability, high sensitivity, strong specificity, and easy operation. It has begun to be applied in the detection of Brucella. The most widely used method is the polymerase chain reaction (PCR) method. Since PCR is a method for detecting nucleic acids, it requires very precise equipment. Generally, the expensive equipment prevents this method from being universally popularised. Therefore, it is necessary to design a convenient, rapid and economical diagnosis method[10]. Enzyme-linked immunosorbent assay (ELISA), the rose bengal test (RBT) and standard tube agglutination test (SAT) can discover the existence of Brucella infection by detecting antibodies in the serum[11]. However, methods have some disadvantages, like false positive and false negative results. Compared with other diagnostic methods, the outstanding advantages of ELISA are higher sensitivity, specificity, and simple operation. Therefore, it has become one of the most widely used detection methods.

When performing ELISA tests, the choice of antigen is important if you want to achieve higher accuracy. Compared with other antigenic molecules of Brucella, lipopolysaccharide (LPS) of smooth Brucella genus is the major virulence determinant currently known, and infected animals are prone to produce antibodies against it[12]. Therefore, LPS is the most commonly used antigen in the serological diagnosis of brucellosis. However, serum diagnostic tests for smooth Brucella LPS as a diagnostic antigen cannot distinguish infections caused by those cross-reactive species[13], examples are Yersinia enterocolitica 09, Vibrio cholerae, Escherichia, and Salmonella, those bacteria often lead to false positive. Many studies have proved that the Brucella outer membrane proteins (OMPs) have strong immunoreactive, and are suitable for the diagnosis of brucellosis as a substitute for LPS, mainly including OMP16, periplasmic protein 26 (BP26), OMP2b[7], and OMP31 [7, 14-16]. False positive results from cross-reactive antibodies can be reduced by using OMPs to replace previous diagnostic antigens.

Although some previous studies have used Brucella OMPs to diagnose brucellosis and achieved good experimental results, there are still many deficiencies that need to be improved. In this research, we used some bioinformatics tools to predict the B and T cell epitopes of the four Brucella OMPs mentioned above. Immediately afterwards, we designed a recombinant multiepitope protein (rMEP) and evaluated this protein as a serum diagnostic antigen for the detection of goat and bovine brucellosis.

\section{Results}

\section{Prediction of B cell epitopes}

The amino acid sequences of four outer membrane proteins were obtained from NCBI database to predict the epitopes of B and T cells. To obtain the most immunogenic B and T cell epitopes, three epitope 
prediction software (ABCPred, BepPred, and COBEPro) and the T Cell Epitope Prediction Tool at IEDB (http://tools.iedb.org/main/tcell/) were applied. Among those epitopes obtained by three epitope prediction software, we selected 25 overlapping epitopes as candidate epitopes (see Table S1). BLASTP results also revealed that the selected epitopes are highly conserved in Brucella.

\section{Production And Purifification Of The Recombinant Protein}

\section{Results}

Linkers are used to link predicted epitopes to avoid the formation of hinge regions (see Fig. 1). Codon optimisation of the prokaryotic expression system was performed according to the amino acid sequence of the constructed fusion protein(see Fig. 2). The synthetic gene was ligated to the expression vector pET28b, the recombinant vector was transformed into E. coli BL21 (DE3) cells in LB medium, and the cells were induced to express. The induced products were analysed on SDS-PAGE(see Fig. 3A ). Ni-NTA affinity was used to purify the recombinant protein, and the purified protein was analysed by SDS-PAGE again (see Fig. 3B ). The concentration of rMEP protein solution is $1.39 \mathrm{mg} / \mathrm{mL}$ in $1 \mathrm{~mL}$.

\section{Serodiagnostic evaluation of rMEP by indirect ELISA}

To assess the diagnostic potential of the recombinant protein studied in this experiment, 159 goat serum samples were tested using indirect ELISA, including 93 cases of brucellosis and 66 cases of nonbrucellosis. A dotplot summarises the OD values of the selected samples in this study(see Fig. 4A). The optimal sensitivity and specificity were obtained by ROC analysis(see Fig. 4B). and according to it, we can get the AUC of this experiment was 0.9976 (95\% confidence interval (Cl), 0.9930 to 1.002), the optimal cut-off value was 0.5955 (calculated by the Youden index), the diagnostic sensitivity was $96.97 \%(95 \% \mathrm{Cl}$, $89.48 \sim 99.63)$, and the specificity was $98.92 \%(95 \% \mathrm{Cl}, 94.15 \sim 99.97)$. At this cut-off, 92 of the 93 positive cases were correctly diagnosed as positive, while only 1 were wrongly diagnosed as negative. 64 negative cases were correctly diagnosed as negative, and only 2 were wrongly diagnosed as positive. Compared with SAT and RBPT, the positive and negative diagnostic accuracy of the ELISA was $98.92 \%$ (92/93) and 96.97\%(64/66), respectively. Moreover, we used LPS as the diagnostic antigen of brucellosis for ELISA detection. The obtained data were also analyzed in the same way as rMEP, the dotplot diagram (see Fig. 4C) and ROC curve (see Fig. 4D) were used to present the results. According to ROC analysis, the AUC was $0.9945(95 \% \mathrm{Cl}, 0.9874 \sim 1.002)$, the optimal cut-off value was 0.7165 , the diagnostic sensitivity was $100 \%(95 \% \mathrm{Cl}, 94.56 \sim 100.0)$, and the specificity was $95.7 \%(95 \% \mathrm{Cl}, 89.35 \sim 98.82)$. At this cut-off value, there were only 4 cases misdiagnosed as positive.

Furthermore, 153 bovine serum samples were tested using rMEP, including 74 cases of brucellosis and 79 cases of non-brucellosis. The dotplot summarises the OD values and optimal sensitivity and specificity obtained by ROC analysis (see Fig. 5A, 5B ). The AUC was 0.9974 ( $95 \% \mathrm{Cl}, 0.9938$ to 1.001), the optimal cut-off value was 0.7725 , the diagnostic sensitivity was $98.65 \%(95 \% \mathrm{Cl}, 92.70 \sim 99.97)$, and the specificity was $96.20 \%(95 \% \mathrm{Cl}, 89.30 \sim 99.21)$. At this cut-off, 73 of the 74 positive cases were correctly diagnosed as positive, while only 1 were wrongly diagnosed as negative. 76 were correctly diagnosed as 
negative, and 3 were wrongly diagnosed as positive. Compared with SAT and RBPT, the positive and negative diagnostic accuracy of the ELISA was $98.65 \%(73 / 74)$ and $96.20 \%(76 / 79)$, respectively. When the LPS was used as the diagnostic antigen, the dotplot diagram (see Fig. $5 \mathrm{C}$ ) and ROC curve (see Fig. 5D) were used to present the results. According to ROC analysis, the AUC was $0.9869(95 \% \mathrm{Cl}, 0.9727$ $\sim 1.001)$, the optimal cut-off value was 0.9695 , the diagnostic sensitivity was $95.65 \%(95 \% \mathrm{Cl}, 87.82$ 99.09), and the specificity was $97.47 \%(95 \% \mathrm{Cl}, 91.15 \sim 99.69)$. At this cut-off value, there were 3 cases misdiagnosed as negative and 2 cases misdiagnosed as positive.

Finally, we designed a cross table (see Table 1) to show the number of positive and negative samples at different cutoff values.

Table 1

Positive and negative predictive values of the test calculated for different cutoff values.

\begin{tabular}{|c|c|c|c|c|c|c|c|}
\hline \multirow[t]{2}{*}{ Cutoff value } & \multicolumn{2}{|c|}{ Positive } & \multicolumn{3}{|c|}{ Negative } & \multirow[t]{2}{*}{ PPV(\%) } & \multirow[t]{2}{*}{ NPV(\%) } \\
\hline & TP & FN & $\mathrm{TI}$ & & & & \\
\hline $0.5955^{a}$ & 92 & 1 & 64 & 2 & 97.87 & 98.46 & \\
\hline $0.7165^{b}$ & 93 & 0 & 62 & 4 & 95.88 & 100.0 & \\
\hline $0.7725^{c}$ & 73 & 1 & 76 & 3 & 96.05 & 98.70 & \\
\hline $0.9695^{d}$ & 71 & 3 & 77 & 2 & 97.26 & 96.25 & \\
\hline \multicolumn{8}{|c|}{ TP, true positives; TN, true negatives; FP, false positives; FN, false negatives; } \\
\hline \multicolumn{8}{|c|}{ PPV, positive predictive value $(\mathrm{TP} /(\mathrm{TP}+\mathrm{FP})) \times 100$} \\
\hline \multicolumn{8}{|c|}{ NPV, negative predictivevalue $(T N /(T N+F N)) \times 100$} \\
\hline \multicolumn{8}{|c|}{${ }^{a}$ cutoff value is calculated by rMEP in goat } \\
\hline \multicolumn{8}{|c|}{${ }^{b}$ cutoff value is calculated by LPS in goat } \\
\hline \multicolumn{8}{|c|}{${ }^{c}$ cutoff value is calculated by rMEP in goat } \\
\hline${ }^{d}$ cutoff value & s calc & latec & LPS & & & & \\
\hline
\end{tabular}

\section{Discussion}

Brucellosis is an easily neglected zoonosis caused by Brucella. It is widely popular worldwide. Especially in endemic areas, the establishment of rapid and accurate diagnostic methods is a prerequisite for 
disease prevention and control. Detection of antibodies using serological methods is often used to diagnose brucellosis in animals. Since B-cell epitopes are molecular sites recognized by antibodies[17], we use a web server and bioinformatics tools to predict the dominant epitopes of Brucella outer membrane proteins. Analysis of the characteristics of the amino acids that make up the four OMPs, including immunological information analysis and prediction of possible B cell epitopes, such as hydrophilicity, surface accessibility and $\beta$-turn angle[18-20]. However, the success rate of predicting immunogenic B-cell epitopes using a single bioinformatics tool is low. Therefore, we used three different prediction software programs (ABCPred, BepiPred, and COBEPro) and T Cell Epitope Prediction Tools to predict the predominant epitopes with immunogenicity. A variety of OMPs from Brucella have been proven to be highly immunoreactive. It has been reported that Brucella OMP antigens can be used for serological diagnosis of diseases and overcome the problem of cross-reactivity of LPS-based serological diagnostic methods. Many studies have performed ELISA tests on brucellosis OMP antigens such as OMP10, OMP19, OMP25, OMP28, and OMP31. These studies show that the use of OMP from Brucella cells is very effective for serological diagnosis of the disease. In this research, we selected four OMPs (BP26, OMP16, OMP31 and OMP2b), and obtained 25 candidate epitopes using three bioinformatics tools and a web server.

At present, there are many methods for diagnosing brucellosis in animals, but there are still some disadvantages such as difficult to operate, and it takes a long time. ELISA is faster and more convenient than other methods and has higher sensitivity and specificity[21], which is consistent with the goal of this work. In our work, rMEP was designed using the selected superior B- and T- cell epitopes as a diagnostic antigen, and indirect ELISA was used to diagnose diseased and healthy serum. Subsequently, we performed ROC analysis to verify the validity of this test, and we got an AUC value of 0.9789 . Compared with the LPS antigen, our rMEP has high accuracy of diagnosis is almost comparable to distinguish Brucella diseased animals from healthy animals.

\section{Conclusions}

In summary, this study shows that the indirect ELISA detection method based on the newly designed rMEP has high sensitivity and specificity, and can be used to detect Brucella in animals. In our study, the E. coli expression system was used to overexpress the recombinant protein consisting of four major OMPs from Brucella and confirmed that it could diagnose brucellosis in animals. This meets the need to produce a large number of diagnostic antigens in a short time, saves time and avoids the preparation of Brucella LPS antigens. In addition, this test method based on rMEP for brucellosis can provide ideas for the diagnosis of other infectious diseases. However, the diagnostic method cannot distinguish the type of Brucella affected. The diagnostic value of this method for different types of Brucella infection is unknown, and in order to make the study more significant, further study is needed to differentiate between vaccinated and non-vaccinated animals.

\section{Methods}




\section{Selection of outer membrane proteins}

After reading a large number of literature on the study of Brucella OMPs, the OMPs that have been proved to have antigenicity and exist in different species of Brucella were selected as the study proteins. Use the website https://www.ncbi.nlm.nih.gov/protein/ to obtain the amino acid sequence of the selected OMPs. In order to avoid cross-reactions with other bacteria, we used the BLASP tool

(https://blast.ncbi.nlm.nih.gov/Blast.cgi) to carry out sequence comparison analysis of amino acids, and selected the conserved amino acid sequence in Brucella according to the comparison results.

\section{Epitope Prediction Of Outer Membrane Proteins}

ABCpred(http://www.imtech.res.in/raghava/abcpred/)[22,.23],

Bepipred(http://www.cbs.dtu.dk/services/Bepipred/0)[24], and COBEpro

(http://scratch.proteomics.ics.uci. edu/)[25] were used for linear B-cell epitope prediction, and overlapping B-cell epitopes were selected as candidate B epitope. T cell epitope were predicted by T Cell Epitope Prediction Tools at IEDB (http://tools.iedb.org/main/tcell/).

\section{Construction, Expression And Purification Of rMEP}

We used the linker peptide GGGS to tandem candidate B and T cell epitopes, infer codons according to the obtained amino acid sequence, and used the optimisation website (http://www.jcat.de/) to perform codon optimisation of the prokaryotic expression system. The Optimized sequence was transferred to Sangon Biotech (Shanghai, China) for gene synthesis. The coding sequences of six His tags was added to the 3 'end of the gene for subsequent purification and identification.

The gene fragment was ligated with the expression vector pGEM-T, and the newly constructed was transformed into E. coli BL21 (DE3) cells which were grown in LB medium. It was the suitable time for us to induce recombinant protein expression when the optical density values at $600 \mathrm{~nm}\left(\mathrm{OD}_{600}\right)$ reached to 0.6. the expression was induced by $0.5 \mathrm{mM}$ isopropyl- $\beta$-D-thiogalactoside (IPTG) and continuted to be incubated for $4 \mathrm{~h}$ at $37 \mathrm{C}$. Bacterial Cells were collected by centrifugation and analysed on SDS-PAGE. The rMEP was purified by nickel agarose affinity chromatography.

\section{Serum Samples And Ethics}

93 cases of goat serum and 74 cases of bovine serum confirmed for brucellosis by both serum tube agglutination test (SAT) and the Rose Bengal plate agglutination test (RBPT) were used as positive samples. 66 goat and 79 bovine serums which were negative for RBPT and SAT were collected as negative samples. All samples were provided by China Animal Health And Epidemiology Center (Qingdao, China). All experiments involves animals or animal samples were fully compliant with an ethical approval granted by the Animal Care and Ethics Committee of Xuzhou Medical University.

\section{ELISA Analysis}


The obtained goat and bovine serum samples were detected by indirect ELISA in which rMEP was used as the diagnostic antigen. The purified rMEP was diluted with PBS buffer solution (0.01 M PBS, pH 7.4) and coated in 96-well immunoassay plates (Corning, USA) at $1 \mu \mathrm{g}$ per well overnight at $4{ }^{\circ} \mathrm{C}$. Each well was added with phosphate buffered saline-Tween 20 (PBST), washed four times, and then blocked with $1 \%$ ovalbumin (OVA, TCl, Japan) for $1.5 \mathrm{~h}$ at $37^{\circ} \mathrm{C}$, washed four times again and incubated with goat and bovine serum at $37 \mathrm{C}$ at a dilution of 1:400 for $1 \mathrm{~h}$. Continuing to wash the plates and incubating the plates with HRP-conjugated recombinant protein G (dilution of 1: 5,000) (thermo, USA) for 25 min at room temperature. Washing the plates, adding $100 \mu \mathrm{L}$ of substrate solution containing TMB (trimethylbenzene) to each well, and placing the plates in the dark at room temperature. After $15 \mathrm{~min}$ of color reaction, $2 \mathrm{M}$ $\mathrm{H}_{2} \mathrm{SO}_{4}$ was added to stop it. Optical density values were gained at $450 \mathrm{~nm}\left(\mathrm{OD}_{450}\right)$ in an ELISA plate reader (BioTek). All samples were measured twice. We evaluated the effectiveness of rMEP indirect ELISA by comparing with PBST and SAT.

In addition, in order to evaluate the detection utility of rMEP, the ELISA test was performed again with lipopolysaccharide (LPS, $0.1 \mu \mathrm{g}$ per well, provided by China Animal Health And Epidemiology Center) antigen, and the two results were compared and analyzed.

\section{Statistical analysis}

GraphPad Prism version 6.05 for Windows was used to obtain the dotplot and receiver-operating characteristic (ROC).

\section{List Of Abbreviations}

AUC: area under the ROC curve; BP26: periplasmic protein 26; ELISA: enzyme-linked immunosorbent assay; LPS: lipopolysaccharide; OMPs: outer membrane proteins; PCR: polymerase chain reaction; RBT: rose bengal test; rMEP: recombinant multiepitope protein; ROC: receiver-operating characteristic; SAT: standard tube agglutination test

\section{Declarations}

\section{Ethics approval}

All experiments involves animals or animal samples were fully compliant with an ethical approval granted by the Animal Care and Ethics Committee of Xuzhou Medical University.

\section{Consent for publication}

Not applicable

\section{Availability of data and materials}

All relevant data in this study are available from the corresponding author upon reasonable request. 


\section{Competing interests}

The authors declare that they have no competing interests.

\section{Funding}

This work was supported by the Natural Science Foundation of the Jiangsu Higher Education Institutions of China (Grant number 18KJB230005) \& the National Natural Science Foundation of China (Grant number 81802101$)$. The funders had no role in study design, data collection and analysis, decision to publish, or preparation of the manuscript.

\section{Authors' contributions}

D.H.Y. and Q.Q.B. analyzed the data and drafted the manuscript. Q.Q.B. performed the assays. Z.J.P. reviewed and made improvements in the manuscript. D.H.Y., H.L. and Z.J.P. conceived and designed the study. All authors read and approved the final version of the paper.

\section{Acknowledgements}

Not applicable

\section{References}

1. Mahmood R, Ali T, Waheed U, Asif M, Khan QM. Application of Serum Based PCR and Fluorescence Polarization Assay for Diagnosis of Brucellosis among People Occupationally at Risk to Disease. Int J Agric Biol. 2016; 18: 377-84.

2. Afley P, Dohre SK, Prasad GB, Kumar S. Prediction of T cell epitopes of Brucella abortus and evaluation of their protective role in mice. Appl Microbiol Biotechnol. 2015; 99:7625-37.

3. Gusi AM, Bertu WJ, Jesús de Miguel M, Dieste-Pérez L, Smits HL, Ocholi RA, et al. Comparative performance of lateral flow immunochromatography, iELISA and Rose Bengal tests for the diagnosis of cattle, sheep, goat and swine brucellosis. PLoS Negl Trop Dis. 2019;13:e0007509.

4. Koyuncu I, Kocyigit A, Ozer A, Selek S, Kirmit A, Karsen H. Diagnostic potential of Brucella melitensis Rev1 native Omp28 precursor in human brucellosis. Cent Eur J Immunol. 2018;43:81-9.

5. Bundle DR, McGiven J. Brucellosis: Improved Diagnostics and Vaccine Insights from Synthetic Glycans. Acc Chem Res. 2017; 50: 2958-67.

6. Ran X, Chen X, Wang M, Cheng J, Ni H, Zhang XX, et al. Brucellosis seroprevalence in ovine and caprine flocks in China during 2000-2018: a systematic review and meta-analysis. BMC Vet Res. 2018;14:393.

7. Vatankhah M, Beheshti N, Mirkalantari S, Khoramabadi N, Aghababa H, Mahdavi M. Recombinant Omp2b antigen-based ELISA is an efficient tool for specific serodiagnosis of animal brucellosis. Braz J Microbiol. 2019; 50:979-84. 
8. Leya M, Kim WK, Cho JS, Yu EC, Kim YJ, Yeo Y, et al. Vaccination of goats with a combination Salmonella vector expressing four Brucella antigens (BLS, PrpA, Omp19, and SOD) confers protection against Brucella abortus infection. J Vet Sci. 2018; 19:643-52.

9. Aragón-Aranda B, de Miguel MJ, Martínez-Gómez E, Zúñiga-Ripa A, Salvador-Bescós M, Moriyón I, et al. Rev1 wbdR tagged vaccines against Brucella ovis. Vet Res. 2019; 50:95.

10. Shi F, Sun Y, Wu Y, Zhu M, Feng D, Zhang R, et al. A novel, rapid and simple method for detecting brucellosis based on rapid vertical flow technology. J Appl Microbiol. 2020; 128:794-802.

11. İrvem A, Yücel FM, Aksaray S, Bor E. Comparison of a new and rapid method, Brucella Coombs gel test with the other methods in the serological diagnosis of brucellosis. Mikrobiyol Bul. 2015; 49:1817.

12. Chaudhuri P, Prasad R, Kumar V, Gangaplara A. Recombinant OMP28 antigen-based indirect ELISA for serodiagnosis of bovine brucellosis. Mol Cell Probe. 2010; 24:142-5.

13. Yagupsky P, Morata P, Colmenero JD. Laboratory Diagnosis of Human Brucellosis. Clin Microbiol Rev. 2019; 33:e00073-19.

14. Alizadeh H, Dezfulian M, Rahnema M, Fallah J, Esmaeili D. Protection of BALB/c mice against pathogenic Brucella abortus and Brucella melitensis by vaccination with recombinant Omp16. Iran J Basic Med Sci. 2019; 22:1302-7.

15. Qiu J, Wang W, Wu J, Zhang H, Wang Y, Qiao J, et al. Characterization of periplasmic protein BP26 epitopes of Brucella melitensis reacting with murine monoclonal and sheep antibodies. PLoS One. 2012; 7:e34246.

16. Shojaei M, Tahmoorespur M, Soltani M, Sekhavati MH. Immunogenicity evaluation of plasmids encoding Brucella melitensis Omp25 and Omp31 antigens in BALB/c mice. Iran J Basic Med Sci. 2018; 21:957-64.

17. Berger P, Lapthorn AJ. The molecular relationship between antigenic domains and epitopes on hCG. Mol Immunol.2016; 76:134-45.

18. Mishra A, Jain A, Arora N. Mapping B-cell epitopes of major and minor peanut allergens and identifying residues contributing to IgE binding. J Sci Food Agr. 2016; 96, 539-47.

19. Petersen B, Petersen TN, Andersen P, Nielsen M, Lundegaard C. A generic method for assignment of reliability scores applied to solvent accessibility predictions. BMC Struct Biol. 2009;9:51.

20. Xie M, Schowen RL. Secondary Structure and Protein Deamidation. J Pharm Sci. 1999; 88:8-13.

21. Aydin S. A short history, principles, and types of ELISA, and our laboratory experience with peptide/protein analyses using ELISA. Peptides. 2015;72:4-15.

22. Xie Q, He X, Yang F, Liu X, Li Y, Liu Y, et al. Analysis of the Genome Sequence and Prediction of B-Cell Epitopes of the Envelope Protein of Middle East Respiratory Syndrome-Coronavirus. IEEE/ACM Trans Comput Biol Bioinform. 2018; 15:1344-50.

23. Chauhan V, Singh MP, Ratho RK. Identification of T cell and B cell epitopes against Indian HCVgenotype-3a for vaccine development- An in silico analysis. Biologicals. 2018; 53:63-71. 
24. Jespersen MC, Peters B, Nielsen M, Marcatili P. BepiPred-2.0: improving sequence-based B-cell epitope prediction using conformational epitopes. Nucleic Acids Res. 2017;45:W24-W29.

25. Sweredoski MJ, Baldi P. COBEpro: a novel system for predicting continuous B-cell epitopes. Protein Eng Des Sel. 2009; 22:113-20.

\title{
Figures
}

\begin{abstract}
BP26
OMP31

OMP16

$\mathrm{OMP} 2 \mathrm{~b}$

1 FLAASFSTIG GGSFAQENQM TTQPARIAVT GGGGSKKAGI EDRDLQTGGI NIQPIYVYPD

61 DKNNLKEPTI TGYGGGSGVN QGGDLNLVND NPSAVINGGG SEARKRAVAN AIAKAKTGGG 121SPMPMPIARG QFGGGSAAAP DNSVGGGSPI AAGENSYNVS VNVVFEIGGG SLAAMFATSA 181MAADVVVSEP SAPGGGSTAA PVDTFSWGGG SFSSFDKEDN EQVSGSLDVG GGSSSVTGSI 241SAGASGLEGK AETKGGGSAY GKVKSAFNLG DDASALHTWS DKGGGSTKAG WTLGAGGGGS 301QSIARSPIAI ALFMSLAVGG GSQYSITIEG HADERGTREG GGSASRGVPT NRMRTISYGN 361ERPVAVGGGS GSAAALVAAS GAQAADGGGS TCLRVHGYVG GGSDVKGGDD VYSGTDRNGW 421DKGGGSALEQ GGDNDGGYTG TTNGGGSFSV WLQGAYGGGS YSSAATPDQN YGQWGGDWAG 481GGSFNLQAAH DDWGKTAVTA NGGGSTVTPE VSYTKFGGEW KDTVAEDNAW GG
\end{abstract}

\section{Figure 1}

Amino acid sequence of rMEP with "GGGS" as the linker 


\section{CATATG}

TTTCTGGCAGCGAGCTTCAGCACCATCGGTGGCGGTTCTTTCGCTCAGGAGAACCAGATGACCAC CCAGCCTGCACGTATCGCAGTAACGGGTGGCGGTTCTAAGAAAGCGGGTATCGAGGACCGTGACC TGCAGACTGGTGGTATCAACATCCAGCCGATCTACGTGTACCCAGACGACAAGAACAACCTGAAA GAGCCGACCATCACCGGTTATGGTGGCGGCTCCGGTGTCAACCAGGGTGGCGACCTGAACCTGGT GAACGACAACCCGAGCGCTGTCATCAACGAGGCGCGCAAACGCGCAGTGGCAAACGCTATCGCT AAAGCAAAGACCGGCGGCGGCTCTCCTATGCCAATGCCAATCGCACGTGGTCAGTTCGGCGGTGG TTCTGCAGCTGCTCCGGATAATTCTGTCCCGATCGCAGCAGGTGAGAACTCTTACAACGTCTCCGT GAACGTGGTGTTCGAAATCGGCGGTGGCTCCATCGCGGCTATGTTCGCAACGTCTGCAATGGCCG CAGACGTGGTGGTATCTGAACCGTCTGCACCGACTGCAGCTCCGGTAGATACTTTCTCCTGGGGC GGTGGTTCCTTCTCTTCCTTCGACAAAGAAGACAACGAACAGGTGAGCGGTAGCCTGGACGTTG GTGGCGGTAGCAGCTCCGTAACCGGTTCCATTAGCGCCGGTGCCTCTGGTCTGGAAGGTAAAGCT GAAACCAAAGGTGGCGGTTCCGCCTACGGTAAAGTTAAATCCGCGTTCAACCTGGGCGACGACG CGTCCGCGCTGCACACCTGGTCCGACAAGACGAAAGCTGGTTGGACCCTGGGTGCGGGTGGCGG СTCTCAATCTATTGCTCGTAGCCCGATTGCTATTGCGCTGTTCATGAGCCTGGCGGTTGGTGGCGGC AGCCAATACAGCATTACCATTGAAGGTCACGCCGACGAACGCGGTACCCGTGAAGGCGGCGGCA GCGCTTCTCGTGGTGTTCCGACTAACCGTATGCGTACCATTTCCTACGGTAACGAACGCCCGGTTG CGGTTGGCGGCGGTTCTGGTTCCGCTGCCGCCCTGGTAGCTGCTTCTGGTGCTCAAGCTGCGGAT GGCGGTGGCTCTACTTGCCTGCGTGTTCATGGTTATGTTGGCGGTGGTAGCGATGTTAAAGGTGGC GACGACGTATACTCCGGTACTGATCGCAACGGTTGGGATAAAGGCGGCGGCTCCGCGCTGGAACA GGGTGGCGATAATGATGGTGGTTACACCGGTACTACGAACGGCGGCGGTTCCTTTTCCGTTTGGCT GCAGGGTGCCTATGGCGGCGGTAGCTATTCTTCTGCCGCGACTCCGGATCAGAATTACGGTCAGTG GGGCGGCGATTGGGCCGGCGGTGGCAGCTTTAATCTGCAGGCGGCGCACGATGATTGGGGCAAA ACTGCGGTTACTGCGAACGGTGGCGGCTCTACCGTAACCCCGGAAGTTTCTTATACCAAATTTGGC GGCGAAT GGAAAGATACCGT T GCGGAAGATAACGCGTGGGGCGGCTAATGA CTCGAG

Figure 2

Codon optimized gene sequence

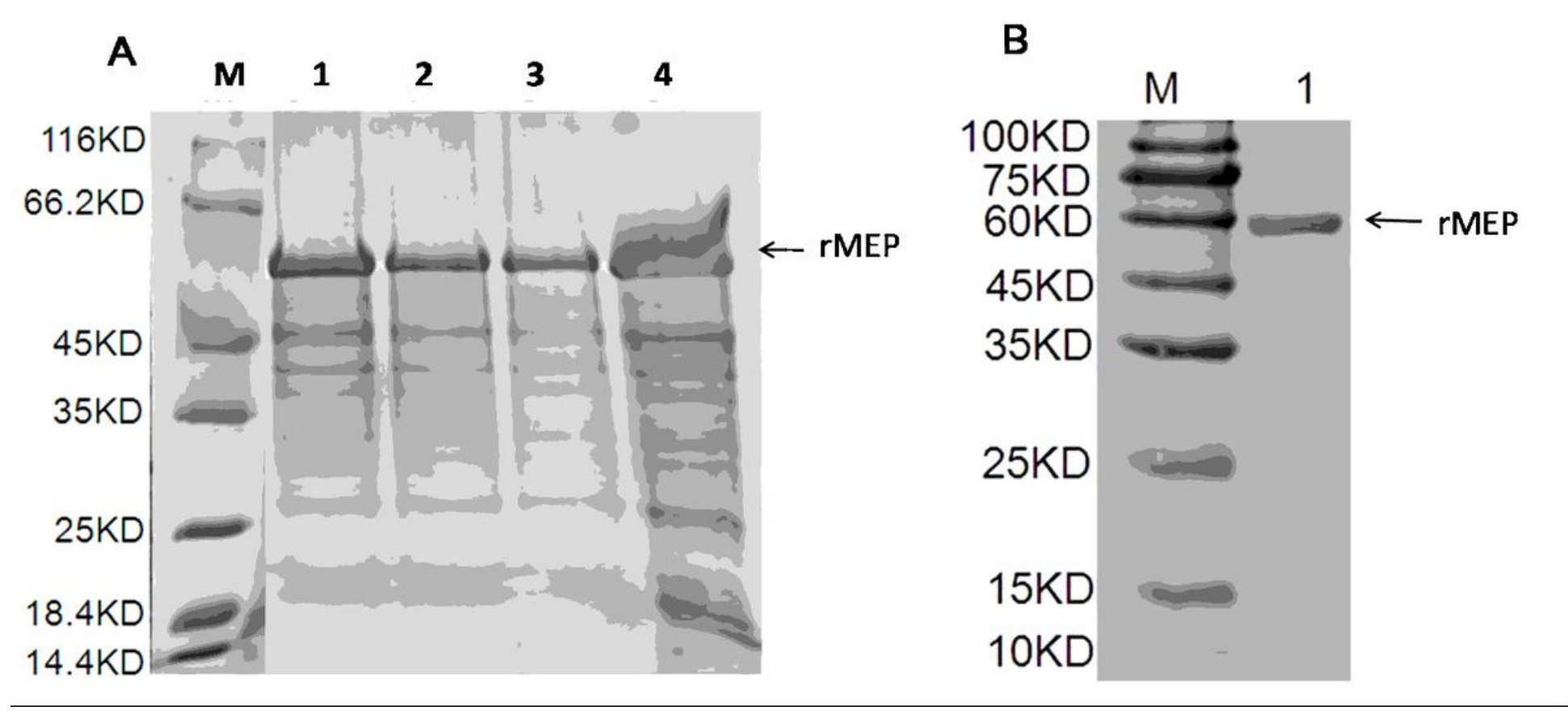


Figure 3

SDS-PAGE analysis of rMEP. (A) Expression analysis of rMEP (M, protein marker; lane 1-4, elution with imidazole). (B) Purification analysis of $\operatorname{rMEP}$ (M, protein marker; Lane 1, purified recombinant protein)
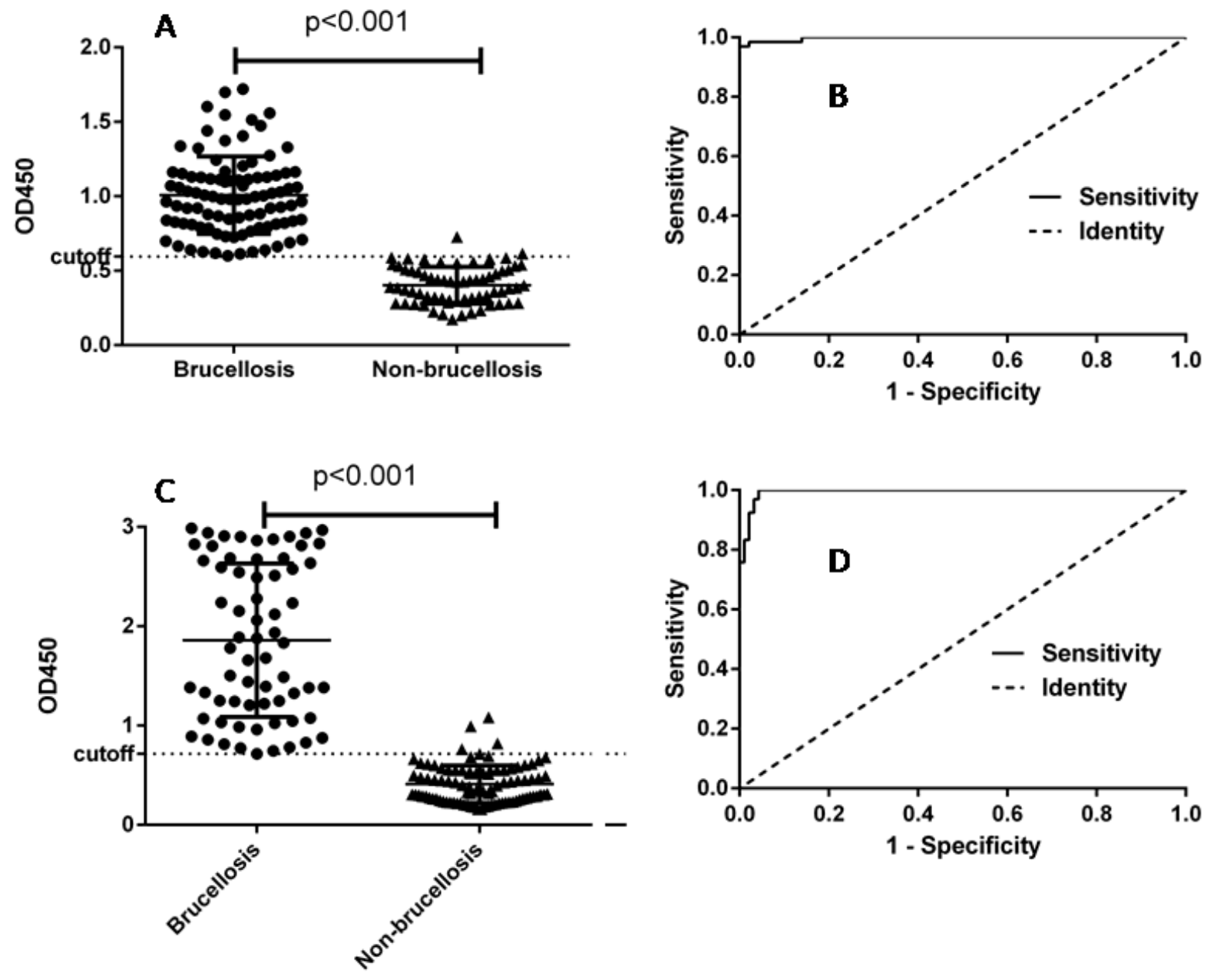

Figure 4

ELISA analysis of goat serum samples. (A) Dotplot of the rMEP ELISA assay. (B) ROC analysis of rMEP IELISA assay results. (C) Dotplot of the LPS antigen ELISA assay. (D) ROC analysis of LPS antigen ELISA assay results. 

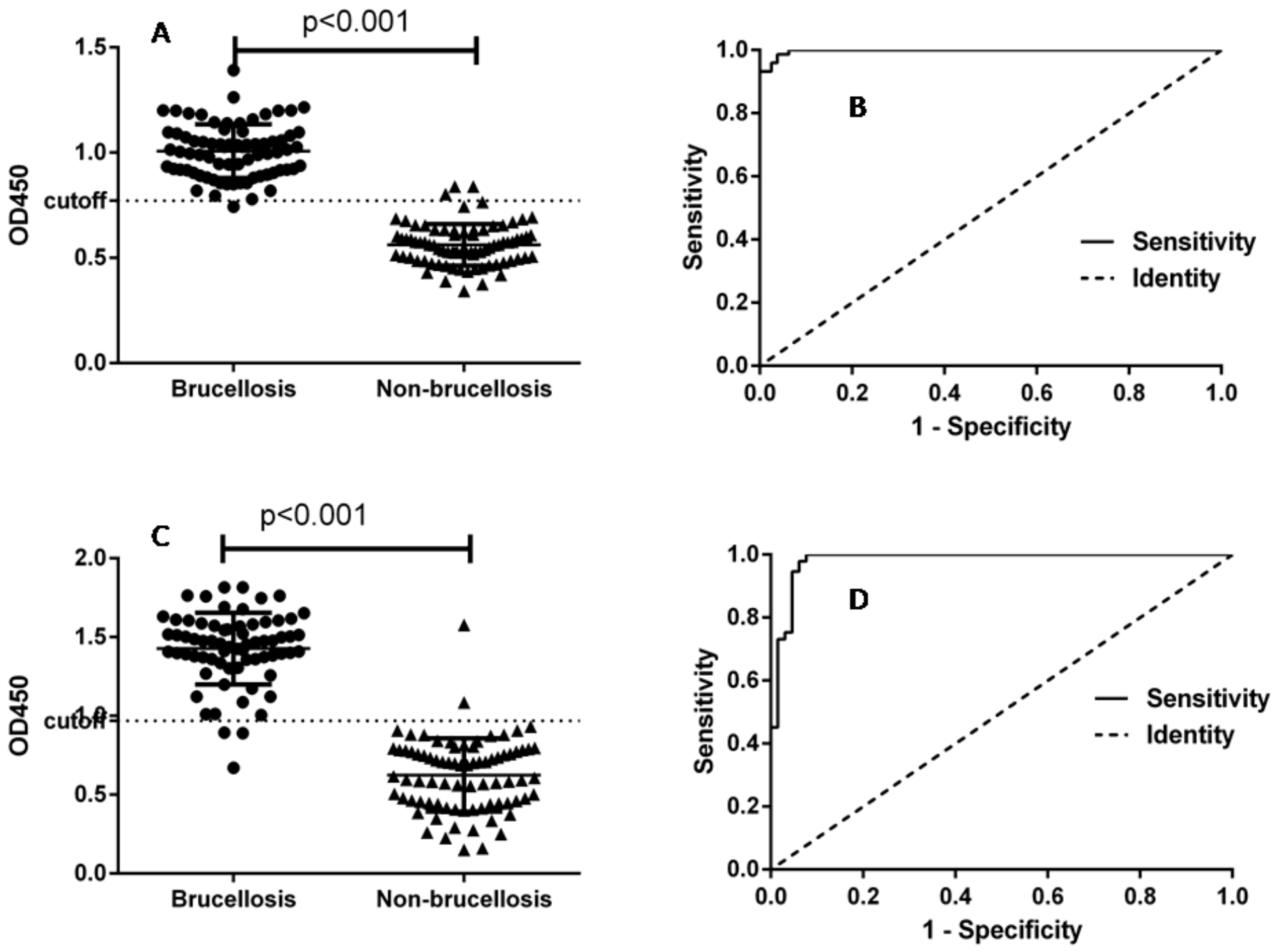

Figure 5

ELISA analysis of bovine serum samples. (A) Dotplot of the rMEP ELISA assay. (B) ROC analysis of rMEP IELISA assay results. (C) Dotplot of the LPS antigen ELISA assay. (D) ROC analysis of LPS antigen ELISA assay results.

\section{Supplementary Files}

This is a list of supplementary files associated with this preprint. Click to download.

- Additionalfiles.docx 